\title{
CENTRALISERS IN WREATH PRODUCTS
}

\author{
by J. D. P. MELDRUM \\ (Received 28th November 1977)
}

In this paper, the centraliser of an arbitrary element of a wreath product is determined. One application of this is to find the breadth of a wreath product (Theorems 21 and 22), a problem which was raised in discussion with Dr. I. D. Macdonald. Another application is to groups generated by elements generating their own centralisers (Theorem 20).

Let $A$ and $B$ be two groups. Define

$$
A^{B}=\{f: B \rightarrow A ; f(b)=e \quad \text { for all but a finite number of elements of } B\}
$$

to be a group by defining the product pointwise

$$
f g(b)=f(b) g(b) \text { for all } b \in B .
$$

Then $A^{B}$ is a restricted direct power of copies of $A$ indexed by elements of $B$. Define $B$ as a group of automorphisms of $A^{B}$ by

$$
f^{b}\left(b_{1}\right)=f\left(b_{1} b^{-1}\right) \text {. }
$$

Then $A$ wr $B$ is the semidirect product of $A^{B}$ by $B$ determined by this definition. A recent paper on wreath products with a good bibliography is $C$. Wells (3).

If $A$ and $B$ are finite $p$-groups, then so is $A$ wr $B$. The class of a finite $p$-group will denote its nilpotency class. The breadth of a finite $p$-group is defined as $b$ where $p^{b}$ is the size of the largest conjugacy class of the group. So $p^{b}$ is the index of the smallest centraliser. If $c$ is the class of the group, there is a conjecture that

$$
b \geqslant c-1 .
$$

A recent paper dealing with this conjecture is Macdonald (1), which has a good bibliography.

Let $f g \in G=A$ wr $B$, where $f \in A^{B}, g \in B$, and let $d h \in C_{G}(f g)$, where $d \in A^{B}$, $h \in B$.

Lemma 1. $d h \in C_{G}(f g)$ if and only if

(i) $h \in C_{B}(g)$,

(ii) $d(x g)=f(x)^{-1} d(x) f(x h)$ for all $x \in B$.

Proof. $d h \in C_{G}(f g)$ if and only if $(d h)^{-1} f g d h=f g$ if and only if $h^{-1} d^{-1} f g d h=f g$ if and only if $d^{-h} f^{h} d^{g^{-1} h} h^{-1} g h=f g$ if and only if $g^{h}=g$ and $d^{-h} f^{h} d^{g^{-1 h}}=f$,

that is

$$
h \in C_{B}(g) \text { and } d^{-1} f d^{g^{-1}}=f^{h-1} .
$$


The latter condition is

$$
d(x)^{-1} f(x) d(x g)=f(x h)
$$

or

$$
d(x g)=f(x)^{-1} d(x) f(x h) .
$$

Corollary 2. If $d h \in C_{G}(f g)$ then

$$
d\left(x g^{n+1}\right)=f\left(x g^{n}\right)^{-1} \ldots f(x)^{-1} d(x) f(x h) \ldots f\left(x h g^{n}\right)
$$

for all $n \geqslant 0$.

Proof. By induction on $n$, using Lemma 1 .

We use $o(g)$ to denote the order of the element $g$ of a group. For reasons that are obvious from Corollary 2 , we will define

$$
\bar{f}(x, g)=f\left(x g^{-n}\right) f\left(x g^{-n+1}\right) \ldots f\left(x g^{m}\right)
$$

where

(i) if $o(g)$ is infinite, then $f\left(x g^{-i}\right)=e$ for $i>n$ and $f\left(x g^{j}\right)=e$ for $j>m$,

(ii) if $o(g)$ is finite, then $n=0$ and $o(g)-1=m$.

Another aspect that will occur several times is that elements of $C_{B}(g)$ will permute the left cosets of $G p\langle g\rangle$ under the right regular representation since $x G p\langle g\rangle h=$ $x h G p\langle g\rangle$. This permutation will be denoted $\rho(h)$.

From now on until further notice, $h$ will always denote an element of $C_{B}(g)$.

Case 1. $o(g)$ is infinite.

In this case $\bar{f}(x, g)$ is uniquely defined for each left coset of $G p\langle g\rangle$. We use $\rho$ as defined above. Then $\rho$ is a homomorphism from $C_{B}(g)$ to the group of permutations of the left cosets of $G p\langle g\rangle$. Since

$$
x h_{1} G p\langle g\rangle=x h_{2} G p\langle g\rangle
$$

if and only if $h_{1} G p\langle g\rangle=h_{2} G p\langle g\rangle$, the kernel of $\rho$ is $G p\langle g\rangle$.

Let $X$ be the set of left cosets of $G p\langle g\rangle$. Partition $X$ into $\left\{X_{i} ; i \in I\right\}$ where

$$
X_{i}=\left\{x G p\langle g\rangle: \bar{f}(x, g)=g_{i}\right\} .
$$

So $X_{i}$ consists of all left cosets of $G p\langle g\rangle$ with a common value for $\bar{f}(x, g)$. Note that $I$ is finite.

Since $\rho\left(C_{B}(g)\right)$ permutes the left cosets of $G p\langle g\rangle$, it permutes the set $\{\bar{f}(x, g)\}$ of values of $\bar{f}(x, g)$ under an obvious extension of the definition of the action of $\rho(h)$. We consider

$$
H(f g)=\left\{h \in C_{B}(g) ; \rho(h) \text { stabilizes } X_{i} \text { for } i \in I\right\} \text {. }
$$

Thus $H(f g)$ consists precisely of those $h \in C_{B}(g)$ such that $\bar{f}(x, g)=\bar{f}(x h, g)$ for all $x \in B$. 
Theorem 3. Let $o(g)$ be infinite. Then $d h \in C_{G}(f g)$ if and only if

(i) $h \in H(f g)$,

(ii) $d\left(x g^{-k+n}\right)=f\left(x g^{-k+n-1}\right)^{-1} \ldots f\left(x g^{-k}\right)^{-1} f\left(x h g^{-k}\right) \ldots f\left(x h g^{-k+n-1}\right)$ for all $n \geqslant 1$ and $k$ is defined by $f\left(x g^{-l}\right)=e=f\left(x h g^{-l}\right)$ for $l>k, d\left(x g^{-l}\right)=e$ if $l \geqslant k$.

Proof. By definition $H(f g) \subseteq C_{B}(g)$. So $h \in C_{B}(g)$. Since $h \in H(f g)$, we have $\bar{f}(x, g)=\bar{f}(x h, g)$. Hence for sufficiently large $n, d\left(x g^{-k+n}\right)=e$. Also for only finitely many left cosets of $G p\langle g\rangle$ do we have $f\left(x g^{i}\right) \neq e$ for any $i$. So $d$ is well defined as an element of $A^{B}$. From (ii), it is obvious that $d(x g)=f(x)^{-1} d(x) f(x h)$ for all $x \in B$. Thus $d h$ satisfies the conditions of Lemma 1 and we have sufficiency.

We now consider necessity. We can assume the results of Lemma 1 and Corollary 2. Let $k$ be defined as in (ii) of Theorem 3. Since $d\left(x g^{-1}\right)=e$ for sufficiently large values of $l$, we can use Corollary 2 and the definition of $k$ to deduce that $d\left(x g^{-l}\right)=e$ for $l \geqslant k$. For sufficiently large values of $n$, we have by definition

$$
\begin{gathered}
\bar{f}(x, g)=f\left(x g^{-k}\right) \ldots f\left(x g^{-k+n-1}\right), \\
\bar{f}(x h, g)=f\left(x h g^{-k}\right) \ldots f\left(x h g^{-k+n-1}\right) .
\end{gathered}
$$

Also by Corollary 2 ,

$$
\begin{aligned}
d\left(x g^{-k+n}\right) & =f\left(x g^{-k+n-1}\right)^{-1} \ldots f\left(x g^{-k}\right)^{-1} d\left(x g^{-k}\right) f\left(x h g^{-k}\right) \ldots f\left(x h g^{-k+n-1}\right) \\
& =\bar{f}(x, g)^{-1} \bar{f}(x h, g)
\end{aligned}
$$

for sufficiently large values of $n$, and this must be $e$ as $d \in A^{B}$. Hence $\bar{f}(x, g)=$ $\bar{f}(x h, g)$. This must hold for all values of $x$. Thus $h \in H(f g)$. We have also shown above that $d\left(x g^{-l}\right)=e$ if $l \geqslant k$ and then the necessity of (ii) follows from Corollary 2 .

Corollary 4. Let $g \in B$ have infinite order. Then $C_{G}(f g)$ is isomorphic to $H(f g)$.

Proof. From Theorem 3, using the map $d h \rightarrow h$ and noting that $d$ is uniquely defined, given $f$ and $h$.

Corollary 5. Let $g \in B$ have infinite order, and satisfy $C_{B}(g)=G p\langle g\rangle$. Then $C_{G}(f g)=G p\langle f g\rangle$.

Proof. Immediate from Corollary 4.

Lemma 6. Let $K \subseteq C_{B}(g)$. Then the orbits of $\rho(K)$ consist of left cosets of $K G p\langle g\rangle$.

Proof. This is verified easily directly from the definition of $\rho$.

Corollary 7. Let $g \in B$ have infinite order. Let $f \in A^{B}$ satisfy $\bar{f}(x, g)=e$ for all $x \in B$. Then $C_{G}(f g)$ is isomorphic to $C_{B}(g)$. 
Proof. This follows immediately from Theorem 3 and Lemma 6.

Corollary 8. Let $g \in B$ have infinite order. Let $f \in A^{B}$ satisfy $|\{\bar{f}(x, g) ; x \in B\}| \geqslant 2$. If $C_{B}(g) / G p\langle g\rangle$ is torsion-free, then $C_{G}(f g)=G p\langle f g\rangle$.

Proof. If $X_{i}=\left\{x G p\langle g\rangle ; \bar{f}(x, g)=g_{i}\right\}$ and $g_{i} \neq e$, then $X_{i}$ is finite since $f \in A^{B}$. So $X_{i}$ cannot satisfy Lemma 6 for any $K \subseteq C_{B}(g)$ such that $K \supset G p\langle g\rangle$ if $C_{B}(g) / G p\langle g\rangle$ is torsion-free. Now apply Corollary 4.

Corollary 9. Let $g \in B$ have infinite order. If $X_{i}=\left\{x G p\langle g\rangle ; \bar{f}(x, g)=g_{i}\right\}$ consists of a single coset of $G p\langle g\rangle$ for some $g_{i}$, then $C_{G}(g)=G p\langle f g\rangle$.

Proof. If $\left|X_{i}\right|=1$, then $X_{i}$ cannot be a union of left cosets of $G p\langle g\rangle$ of the form $x K G p\langle g\rangle$ with $K \supset G p\langle g\rangle$. Now apply Lemma 6 and Corollary 4 .

Corollary 10. Let $B$ have a set of generators of infinite order. Then $G$ can be generated by a set of elements which generate their own centralisers.

Proof. Let $B=G p\left\langle b_{i} ; i \in I, o\left(b_{i}\right)\right.$ is infinite $\rangle$.

By choosing suitable $f_{i j}$ of the type $f_{i j}(x)=e$ for all but $x=b_{i}, f_{i j}\left(b_{i}\right)=a_{j}$, where $a_{j}$ runs through a generating set of $A$, we can apply Corollary 9 to get the result.

There are a number of results along these lines which could be stated. But we will turn to the next case now.

Case 2. $o(g)$ is finite.

Let $o(g)=m$. In this case $\bar{f}(x, g)$ is not uniquely defined for a given coset of $G p\langle g\rangle$. For this case we have that

$$
\bar{f}(x, g)=f(x) f(x g) \ldots f\left(x g^{m-1}\right) .
$$

Note that

$$
\begin{aligned}
\bar{f}(x g, g) & =f(x)^{-1} \bar{f}(x, g) f\left(x g^{m}\right) \\
& =f(x)^{-1} \bar{f}(x, g) f(x) .
\end{aligned}
$$

Lemma 11. Let $g \in B$ have finite order $m$. Let $d h \in C_{G}(f g)$. Then $\bar{f}(x h, g)=$ $d(x)^{-1} \bar{f}(x, g) d(x)$.

Proof. By Corollary 2, and using the fact that $g^{m}=e$,

$$
\begin{aligned}
d(x) & =d\left(x g^{m}\right)=f\left(x g^{m-1}\right)^{-1} \ldots f(x)^{-1} d(x) f(x h) \ldots f\left(x h g^{m-1}\right) \\
& =\vec{f}(x, g)^{-1} d(x) \vec{f}(x h, g)
\end{aligned}
$$

giving the result we want, after a slight rearrangement. 
Define $K(f g)$ by

$$
K(f g)=\left\{h \in C_{B}(g) ; \bar{f}(x h, g) \text { is a conjugate of } \bar{f}(x, g) \text { for all } x \in B\right\} .
$$

Lemma 12. $K(f g)$ is a subgroup of $B$, and $g \in K(f g)$.

Proof. Since $\bar{f}(x g, g)=f(x)^{-1} \bar{f}(x, g) f(x)$ and $g \in C_{B}(g)$, so $g \in K(f g)$ and $K(f g)$ is not empty. Let $h_{1}, h_{2} \in K(f g)$. Suppose

$$
\bar{f}\left(x h_{i}, g\right)=b_{i}(x)^{-1} \bar{f}(x, g) b_{i}(x), i=1,2 .
$$

Then $\bar{f}(x, g)=b_{i}(x) \bar{f}\left(x h_{i}, g\right) b_{i}(x)^{-1}, i=1,2$. So

$$
\begin{aligned}
\bar{f}\left(x h_{1} h_{2}^{-1}, g\right) & =b_{2}\left(x h_{1}\right) \bar{f}\left(x h_{1}, g\right) b_{2}\left(x h_{1}\right)^{-1} \\
& =b_{2}\left(x h_{1}\right) b_{1}(x)^{-1} \bar{f}(x, g) b_{1}(x) b_{2}\left(x h_{1}\right)^{-1} .
\end{aligned}
$$

Hence $h_{1} h_{2}^{-1} \in K(f g)$ and we have proved the lemma.

Lemma 13. Let $g$ have finite order. Let $d h \in C_{G}(f g)$. Then $\{\bar{f}(x, g) ; x \in y G p\langle h\rangle$ for some fixed $y$ \} forms a conjugacy class in $A$.

Proof. Directly from Lemma 11.

Lemma 14. If $\bar{f}(x, g) \neq e$ for some $x \in B$ and $g \in B$ has finite order, then $d h \in$ $C_{G}(f g)$ satisfies $o(h)$ is finite.

Proof. This follows quickly from Lemma 13.

Theorem 15. Let $o(g)$ be finite. Then $d h \in C_{G}(f g)$ if and only if

(i) $h \in K(f g)$,

(ii) $d(x g)=f(x)^{-1} d(x) f(x h)$,

(iii) if $\bar{f}(x, g) \neq e$ for some $x \in B$, then $o(h)$ is finite,

(iv) $\bar{f}(x h, g)=d(x)^{-1} \bar{f}(x, g) d(x)$.

Proof. We prove necessity first. The definition of $K(f g)$ and Lem ma 11 show that (i) and (iv) are necessary. Lemma 14 shows the necessity of (iii). Lemma 1 shows the necessity of (ii).

Since $K(f g) \subseteq C_{B}(g$.), (i) and (iv) give sufficiency by Lemma 1 .

Theorem 15 is just a restatement of earlier results which enables us to specify exactly the elements of $C_{G}(f g)$. Given $f g \in G$, we first determine $K(f g)$, which we know contains $G p\langle g\rangle$. If $\bar{f}(x, g) \neq e$ for some $x \in B$, then we can only choose elements $h$ in $K(f g)$ of finite order. Any power of $g$ is such an element. We can now determine $d \in A^{B}$ such that $d h \in C_{G}(f g)$. Theorem 15 (iv) determines the coset of $C_{A}(\bar{f}(x, g))$ to which $d(x)$ belongs. Then Theorem 15 (ii) determines the values of $d\left(x g^{i}\right)$ for $1 \leqslant i<o(g)$. If $\bar{f}(x, g)=e$ for all $x \in B$, then there is no restriction on the choice of $h$ in $K(f g)$. Note that any possibility of a double definition for $d(x)$ due to Theorem 15 (ii) is taken care of by Theorem 15 (iv) and if $d(x)$ is chosen to satisfy Theorem 15 
(iv), then $d(x g)$ defined by (ii) satisfies

$$
\begin{aligned}
d(x g)^{-1} \bar{f}(x g, g) d(x g) & =f(x h)^{-1} d(x)^{-1} f(x) \vec{f}(x g, g) f(x)^{-1} d(x) f(x h) \\
& =f(x h)^{-1} d(x)^{-1} \bar{f}(x, g) d(x) f(x h) \\
& =f(x h)^{-1} \bar{f}(x h, g) f(x h) \\
& =\bar{f}(x g h, g)
\end{aligned}
$$

namely Theorem 15 (iv) with $x g$ replacing $x$.

Let $X$ be the set of left cosets of $G p\langle g\rangle$ in $B$.

Theorem 16. Let $f g \in G$, and let $o(g)$ be finite. Then there is a homomorphism from $C_{G}(f g)$ onto $K(f g)$ sending $d h \rightarrow h$, whose kernel is isomorphic to $\Pi_{x \in T} C_{A}(\bar{f}(x, g))$, where $T$ is a left transversal of $G p\langle g\rangle$ in $B$ and $\Pi$ denotes restricted direct product.

This result follows from the remarks above. We look at two special cases.

Lemma 17. Let $g \in B$ have finite order. Then $C_{G}(g)$ is isomorphic to $\left(\Pi_{x \in T} A\right) C_{B}(g)$.

This is a well-known result, namely $d h \in C_{G}(g)$ if and only if $h \in C_{B}(g)$ and $d$ is constant on left cosets of $G p\langle g\rangle$.

Lemma 18. Let $e \neq f \in A^{B}$. Then $K(f)$ is a torsion group and $C_{G}(f)$ is isomorphic to $\left(\Pi_{x \in B} C_{A}(f(x))\right) K(f)$.

Proof. This follows directly from Theorems 15 and 16 once we remember that $\bar{f}(x, e)=f(x)$ and $\bar{f}(x, g) \neq e$ for some $x$, since $e \neq f$.

Lemma 19. Let $A$ and $B$ be non-trivial groups. Let $g \in B$ have finite order. Then $C_{G}(f g)>G p\langle f g\rangle$.

Proof. Let $f g$ satisfy $C_{G}(f g)=G p\langle f g\rangle$. Then we must have $K(f g)=G p\langle g\rangle$ by Theorem 16. Also given $d h \in C_{G}(f g), d$ must be uniquely determined by $h$, as $h=g^{i}$ for some $i$ and then $d h=(f g)^{i}$. So the kernel of the homomorphism described in Theorem 16 must be the identity. But this is obviously impossible. If $g=e$, the result follows directly from Lemma 18.

Theorem 20. Let $G=A$ wr $B, A$ and $B$ be non-trivial groups. Then $G$ is generated by a set of elements which generate their own centralisers if and only if $B$ can be generated by a set of elements all of which have infinite order.

Proof. The sufficiency follows from Corollary 10 and the necessity from Lemma 19. 
As an application of this work we consider the breadth of the wreath product of two finite $p$-groups.

We first note that the breadth of a finite $p$-group $G$ is given by $b(G)=b$, where $p^{b}$ is the index of the smallest centraliser in $G$. Let $G=A$ wr $B$ where $A$ and $B$ are finite $p$-groups. By Theorem 16

$$
C_{G}(f g) \cong \prod_{x \in T} C_{A}(\bar{f}(x, g)) \cdot K(f g)
$$

where $f g \in G$. So we seek to make $C_{A}(\bar{f}(x, g)), K(f g)$ and $T$ as small as possible. But there is a conflict between the first and the last two of these.

Let $A$ have order $p^{a}, B$ have order $p^{b}$ and exponent $p^{e}$. Let the breadth of $A$ be $w$.

Theorem 21. Let $G=A$ wr $B$ have constants as defined above. Then the breadth of $G$ is

(i) $a p^{b}-(a-w) p^{b-e}+b-e$

if $A$ has two distinct conjugacy classes of maximal size,

(ii) $a p^{b}-(a-w) p^{b-e}+\max \{y, b-e-x\}$

if $A$ has only one conjugacy class of maximal size, $x$ is defined by $p^{w-x}$ is the size of the second largest conjugacy class in $A, y$ is defined as $p^{b-y}=\min \left(\left|C_{B}(g)\right| ; O(g)=p^{e}\right)$.

Proof. Let $g \in B$ have order $p^{j}$. Then $j \leqslant e$, and the transversal $T$ of $G p\langle g\rangle$ has order $p^{b-j}$.

(i) Suppose $A$ has two distinct conjugacy classes of maximal size. Then choose $f$ such that $\bar{f}(x, g)$ lies in one of these conjugacy classes for all but one of the cosets of $G p\langle g\rangle$, and in the other one for the remaining coset of $G p\langle g\rangle$. This will ensure that $K(f g)=G p\langle g\rangle$. So if we choose $g$ to have maximal order, namely $p^{e}$ we have made $C_{G}(f g)$ as small as possible. Hence the breadth of $G$ is the exponent of

$$
p^{a p^{b+b}} / p^{(a-w) p^{b-e}} \cdot p^{e}=p^{a p^{b-(a-w) p b-e+b-e},}
$$

i.e., the breadth of $A$ is $a p^{b}-(a-w) p^{b-e}+b-e$.

(ii) Suppose $A$ has only one conjugacy class of maximal size, and let $x, y$ be defined as in the statement of the theorem. If we try to follow the same process as in (i), we find that either all the $\bar{f}(x, g)$ lie in the same (maximal) conjugacy class, and then $K(f g)=C_{B}(g)$, or one of $\bar{f}(x, g)$ lies in the second largest maximal class and then $K(f g)=G p\langle g\rangle$. In the first of these cases we get a conjugacy class size

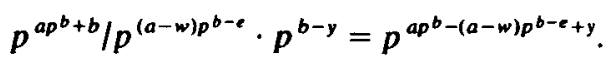

Note that $b-y \geqslant e$, i.e. $b-e \geqslant y$ by definition of $y$. In the second case we get a conjugacy class of size

$$
\begin{gathered}
p^{a p^{b+b}} / p^{(a-w) \times p^{b-e-1)}} \cdot p^{a-w+x} \cdot p^{e} \\
=p^{a p^{b}-(a-w) p^{b-e-x+b-e}} .
\end{gathered}
$$

So the breadth of $G$ is at least

$$
a p^{b}-(a-w) p^{b-e}+\max \{y, b-e-x\} .
$$


If we choose $g$ not to be of maximal order then we would replace $e$ by $j<e$ in the formula, and increase the last term by $\max \left\{e-j, y^{\prime}-y\right\}$, where $p^{b-y^{\prime}}=$ $\min \left(\left|C_{B}(g)\right| ; o(g)=p^{j}\right)$.

The second term would decrease by

$$
(a-w) p^{b-j}-(a-w) p^{b-e}=(a-w) p^{b-e}\left(p^{e-j}-1\right) .
$$

Obviously $e-j \leqslant(a-w) p^{b-c}\left(p^{e-j}-1\right)$. But $b-y^{\prime} \geqslant j$ and so $y^{\prime} \leqslant b-j$. Hence $y^{\prime}-y \leqslant$ $b-j$. It is easy to check that $p^{z}-z \geqslant p^{z-1}$ for positive integral values of $z$. Hence $(a-w) p^{b-j}-\left(y^{\prime}-y\right) \geqslant(a-w) p^{b-j-1}$ and thus

$$
(a-w) p^{b-j}-(a-w) p^{b-e}-\left(y^{\prime}-y\right) \geqslant(a-w) p^{b-j-1}-(a-w) p^{b-e} \geqslant 0 .
$$

So by choosing $g$ not to be of maximal order we get a smaller conjugacy class. This finishes the proof of the theorem.

Theorem 22. Let $A$ be a cyclic group of order $p^{a}, B$ a cyclic group of order $p^{b}$. Then the breadth of $A \mathrm{wr} B$ is equal to the class of $A$ wr $B$ less one if and only if $a=1$ or $b=1$.

Proof. The class of $A$ wr $B$ is $p^{b}+(a-1) p^{b-1}(p-1)$, and the breadth of $A$ wr $B$ is $a p^{b}-a$. Then

$$
\begin{array}{ll}
a p^{b}-a=p^{b}+(a-1) p^{b-1}(p-1)-1 & \text { if and only if } \\
(a-1)\left(p^{b}-p^{b-1}(p-1)\right)=a-1 & \text { if and only if } \\
(a-1) p^{b-1}=a-1 & \text { if and only if } \\
a=1 \text { or } p^{b-1}=1 . &
\end{array}
$$

This gives the result.

The case $a=1$ might have been expected. But the case $b=1$ is somewhat surprising. The class of general wreath products is given by D. Shield (2). To do a precise comparison between this and the breadth of $A$ wr $B$ would involve a good deal of analysis which would not be in character with the rest of this paper.

\section{REFERENCES}

(1) I. D. MacDonald, The breadth of finite p-groups I, Proc. A. Royal Soc. Edinburgh. 78 (1977-78), 31-39.

(2) D. SHIELD, Power and commutator structure of groups, Bull. Austral. Math. Soc. 15 (1976), 315-317.

(3) C. WELls, Some applications of the wreath product construction, American Math. Monthly 83 (1976), 317-338.

UNIVERSITY OF EDINBURGH 